Available online at GSC Online Press Directory

GSC Biological and Pharmaceutical Sciences

e-ISSN: 2581-3250, CODEN (USA): GBPSC2

Journal homepage: https://www.gsconlinepress.com/journals/gscbps

(RESEARCH ARTICLE)

\title{
Effect of genotype on slaughtering performance, blood analyses and meat quality of laying hens reared in different conventional cage densities
}

\author{
Yıldırım Fatih 1, ${ }^{*}$, Özentük Uğur ${ }^{1}$, Özlü Hayrunnisa 2, Urçar Gelen Sevda 3 , Apaydın Yıldırım Betül 4, Atasever \\ Mustafa ${ }^{3}$ and Ylldız Ahmet ${ }^{1}$ \\ ${ }^{1}$ Department of Animal Science, Faculty of Veterinary Medicine, University of Atatürk, Erzurum-25240, Turkey. \\ 2 Department of Nutrition and Dietetics, Faculty of Health Sciences, University of Atatürk, Erzurum-25240, Turkey. \\ ${ }^{3}$ Department of Food Hygiene and Technology, Faculty of Veterinary Medicine, University of Atatürk, Erzurum-25240, \\ Turkey. \\ ${ }^{4}$ Department of Biochemistry, Faculty of Veterinary Medicine, University of Atatürk, Erzurum-25240, Turkey.
}

Publication history: Received on 22 November 2018; revised on 06 December 2018; accepted on 11 December 2018

Article DOI: https://doi.org/10.30574/gscbps.2018.5.3.0141

\begin{abstract}
The aim of the study was to evaluate the effects of genotype on slaughtering performance, blood analyses and meat quality of laying hens reared in different conventional cage densities. Laying hen flocks consisted of 180 Novogen White, 180 ATAK-S and 180 ISA-Brown at 72 weeks old reared in different conventional cage densities $\left(312.50 \mathrm{~cm}^{2} / \mathrm{hen}\right.$ and $468.75 \mathrm{~cm}^{2} / \mathrm{hen}$ ). In the trial, one bird per each replica (9 in replica) were selected from every treatment ( 6 in treatment, 54 birds in total), and their body weights were weighed. Then, they were slaughtered and the carcass traits and some organs were weighed. Also, the blood and breast meat samples were taken for biochemical and meat analyses. In general, the ISA-Brown and ATAK-S strains received higher values than the Novogen White strain in terms of carcass traits. Also, according to stress parameters, it was observed that ISA-Brown strain was more resistant than others in high cage density. Moreover, the bacterial count and meat quality results of hens, the ISA-Brown strain is better than other strains. However, the texture analyses results of the Novogen White strain are better than ISA-Brown and ATAK$\mathrm{S}$ strains. As a result, the meat quality of the laying hens can be affected at different levels depending on the genetic structure of the chickens and the cage density of which they are reared.
\end{abstract}

Keywords: Chicken; Strain; Settlement frequency; Carcass; Texture; Stress

\section{Introduction}

In poultry farming, the number of animals reared per unit area is very important for the profitability of the business. In intensive poultry farming, production is carried out in cage systems. Some of the environmental factors that affect the yield characteristics of animals in cage systems are related to cage properties and cage conditions. Cage density (CD) or settlement frequency is an important stress factor in birds. Sheltering in crowded environments, or reduced cage floor space per animal, affects yield in the negative direction [1]. In conventional laying hen cages (CC), deprivation of physical space and inability to perform highly motivated behaviours causes to stress and inactivity [2].

Many changes that occur during the postmortem muscle metabolism affect the quality of the meat [3]. Meat quality is influenced by agents such as strain, stress and activity, and is determined relatively by measures of $\mathrm{pH}$, tenderness and colour [2]. The usual muscle colour and tenderness values typically occur when final pH ranges between 5.9 and 6.1 [46]. On the other hand, a muscle $\mathrm{pH}$ lower than 5.8 is the link with paler and softer meat. However, a muscle pH greater than 6.2 links with darker and firmer meat [5-7]. The laying chicken meat is generally not preferred for human

\footnotetext{
${ }^{*}$ Corresponding author

E-mail address: fatihyildirim@atauni.edu.tr
} 
consumption [2]. Because, the laying hens have a low meat yield [8], less meat tender than broilers [9], and are also more exposed to splinter during meat processing [10].

In the past century, livestock production has progressively shifted to provide meats of high and consistent eating quality and to obtain high amounts of high-value proteins. And also, the people directed towards fostering a secure and highly convenient meat of consistent eating quality [11]. Among the poultry meat, which plays an important role in satisfying the animal product needs, the meats of broiler strains are generally preferred. However, as alternative meat sources were searched in recent years, in this study, it was tried to obtain alternative chicken meat strains by examining the general meat yield level and consumption status in some strains of laying hens. Thus, it was hypothesized that hens in different cage densities, who had to differ opportunity to locomotory behaviour, would differ improved meat quality among laying hen strains. So, the aim of the current study was to evaluate the effects of genotype on slaughtering performance, blood analyses and meat quality of laying hens which three different genotypes (Novogen White, ATAK-S and ISA-Brown at 72 weeks old) reared in different $\operatorname{CD}\left(312.50 \mathrm{~cm}^{2} /\right.$ hen and $\left.468.75 \mathrm{~cm}^{2} / \mathrm{hen}\right)$.

\section{Material and methods}

\subsection{Animal and experiment design}

This research was carried out in accordance with the guidelines of the Atatürk University Local Board of Ethics Committee for Animal Experiments, which has approved the study protocol of this research (HADYEK decision no: 72). The animals were housed at the Poultry Research Centre of Veterinary Science at the Atatürk University. Laboratory analyses were conducted at the Veterinary Science of Atatürk University. Laying hen flocks consisted of 180 Novogen White, 180 ATAK-S and 180 ISA-Brown (consisting of total 540 hens). The birds that arrived at the facility at 19 weeks were housed at $21^{\circ} \mathrm{C}$ and length of daylight was gradually increased from 13 to $17 \mathrm{~h}$ per d by 27 weeks of age. And, the daily 17-hour daylight period was continued until 72 weeks of age. The CC system consists of totaling 54 CCs, or 18 cages per strain. Each CC measured $62.5 \mathrm{~cm}$ wide by $60 \mathrm{~cm}$ deep and had a height of $46-51 \mathrm{~cm}$. Laying hens were allocated into six treatments ( 3 strain $\times 2$ cage densities) and nine replicates per treatment. At each replicate, eight or twelve birds were used in an environmentally-controlled room. The cage densities consisted of twelve (312.50 $\mathrm{cm}^{2} /$ hen) and eight ( $\left.468.75 \mathrm{~cm}^{2} / \mathrm{hen}\right)$ hens.

\subsection{Processing and sample preparation}

The feed was withdrawn twelve hours before slaughter. At the end of the trial (72 weeks old), one bird per each replica ( 9 in replica) were randomly selected from every treatment ( 6 in treatment, 54 birds in total). After marking and noting that specified treatment and replication's properties, they were weighed and then slaughtered by severing the jugular vein carcass to determine the carcass characteristics. After this applications the feathers, heads, chicken feet and inner organs (except kidneys and lungs) of the chickens were removed. And then the heads, chicken feet, hearts, livers, gizzards, spleens, abdominal fat and hot carcasses were weighed. The carcasses were kept at $+4{ }^{\circ} \mathrm{C}$ for $24 \mathrm{~h}$ and were weighed cold posture; afterward the thighs (from articulatio coxae), wings (from articulatio humeri), breasts (from articulatio sternocostal), neck and back were removed according to regulates and requirements of Institute of Turkish Standards [12]. The organs were weighed with the skin [13]. The head, chicken feet, some internal organs, and carcass were calculated about BW at sacrifice [14].

\subsection{Blood analyses}

The animals included in the study were slaughtered at a slaughterhouse located at a distance of $100 \mathrm{~m}$ to the cages in which they were raised. Therefore, transport stress was eliminated. The blood samples were taken at slaughter, collected into vacuum tubes anticoagulant centrifuged at $3000 \mathrm{rpm}$ for 10 minutes, at $4{ }^{\circ} \mathrm{C}$ and plasma stored at $-20^{\circ} \mathrm{C}$ until laboratory analyses. Oxidative stress is defined as an imbalance between the production of free radicals and reactive metabolites and, it can be determined with malondialdehyde (MDA), glutathione peroxidase (GPx), glutathione (GSH), superoxide dismutase (SOD) and catalase (CAT) measurements [15].

Plasma malondialdehyde (MDA) was analysed by the method of Yoshioka et al. [16], GSH levels were measured according to a method described by Tietze [17], SOD activities were determined using xanthine and nitroblue tetrazolium as the substrates, and were calculated from percent inhibition of formazan production [18]. GPx levels were determined according to methods of Matkovics [19] and CAT activity was determined with a spectrophotometric assay of hydrogen peroxide [20]. All of the biochemical parameters were measured with Biotek ELISA Reader (Bio Tek $\mu$ Quant MQX200 Elisa reader/USA). 


\subsection{Determination of meat quality}

The breast meat samples from the carcasses which had been washed and stored at $4{ }^{\circ} \mathrm{C}$ for $24 \mathrm{~h}$ were cut out. The chicken breast meat samples were placed on polyethylene plates, covered with stretch film. Subsequently, the samples were analysed for $\mathrm{pH}$, thiobarbituric acid reactive substances (TBARS), aw and colour $\mathrm{L}^{*}, \mathrm{a}^{*}, \mathrm{~b}^{*}$ (L*: lightness; $\mathrm{a}^{*}$ : redness; $\mathrm{b}^{*}$ : yellowness) and for total aerobic mesophilic bacteria, total psychrophilic bacteria, Coliforms, Enterobacteriaceae and Staphylococcus aureus counts. The $\mathrm{pH}$ values of the samples were measured as described by Gokalp et al. [21]. Accordingly, $10 \mathrm{~g}$ was added $100 \mathrm{ml}$ of distilled water. After being homogenised with an UltraTurrax device (IKA Werk T 25, Germany) for 1 minute, pH values were measured (WTW Inolab, Germany). The colour intensities $\left(L^{*}, a^{*}, b^{*}\right)$ of the cross-sectional areas of the breast meat samples were determined using a Minolta colorimeter (CR-200, Minolta Co, Osaka, Japan). Colour measurements were performed directly on the surface of muscle tissue, by removing the skin [22].

Water activity (aw) values were measured using the Aqualab 4TE (USA) device. Meat samples were placed in the container of the device for the reading of the aw values. The microbiological analyses of the samples were performed in compliance with the method described by Baumgart et al. [23]. Accordingly, $25 \mathrm{~g}$ of the meat samples were homogenized in $225 \mathrm{~mL}$ of sterile Ringer solution. Subsequently, serial dilutions of the homogenates were prepared. Inoculations were made using the spread plate technique. The TMAB and TPAB counts were determined on Plate Count Agar (PCA, Merck) incubated under aerobic conditions respectively at $30 \pm 1{ }^{\circ} \mathrm{C}$ for $72 \pm 1 \mathrm{~h}$ and $7 \pm 1{ }^{\circ} \mathrm{C}$ for 10 days. For Enterobacteriaceae count, $1 \mathrm{~mL}$ of the appropriate dilutions were seeded on Violet Red Bile Dextrose Agar (VRBDA, Merck), and incubated at $30^{\circ} \mathrm{C}$ under anaerobic conditions for $2 \mathrm{~d}$. The Coliforms count $1 \mathrm{~mL}$ of the appropriate dilutions was seeded on Violet Red Bile Agar (VRBA, Merck), and incubated at $30{ }^{\circ} \mathrm{C}$ under anaerobic conditions for $2 \mathrm{~d}$. Staphylococcus aureus count was determined on baird parker agar (BPA) incubated under aerobic conditions at $30 \pm 1$ ${ }^{\circ} \mathrm{C}$ for $48 \pm 1 \mathrm{~h}$. Bacterial counts were expressed in log cfu g-1 [22].

Texture profile analyses (TPA) was performed by using a TA.XT2 Texture Analyser (Stable Micro Systems, England). Chicken breast meat samples were cut into approximately $2 \mathrm{~cm}$ cubes. The core of the sample was compressed twice to $50 \%$ of their original height using a cylindrical-shaped piston, $6 \mathrm{~mm}$ in diameter. The texture probe was oriented perpendicular to the muscle fibers, and measurements were made at ambient temperature. Texture profile analyses (TPA) parameters of hardness, cohesiveness, adhesiveness, springiness and chewiness were determined [24].

\subsection{Statistical analyses}

The variables concerning slaughtering performance of hens, blood analyses, meat quality and texture profile analyzed completely randomized design using GLM procedure of SPSS 20 (Version 20, IBM Corp., Armonk, NY, USA). The statistical analyses of Tukey multiple range tests and variance were used to compare outcomes in unique groups. The level of significance for statistical differences in all analyses was assessed at $P<0.05$.

\section{Results}

\subsection{Slaughtering performance of hens}

In Table 1, the body weight, some internal organ weights and slaughtering performance of the hens are reported. In treatment combined groups, the body weight and hot-cold carcass, feet, thigh, breast, wing, abdominal fat percentages of ISA-Brown and ATAK-S hens were heavier than the Novogen White hens. Besides, the head's percentages of hens were higher in Novogen White than the ATAK-S and ISA-Brown hens in treatment combined $(P<0.001)$. But, the other heart, liver, gizzard, spleen, neck and back percentages of hens were not found a significant difference among the treatment combined $(P>0.05)$. In strains combined groups, the feet and wing percentages of hens in CD- 2 were higher than CD-1 $(P<0.05)$, and this situation was statistically significant. But, statistically, the other strain combined variables of hens were insignificant $(P>0.05)$. In addition, when the significant different between CD-1 and CD-2 in itself of the strains were examined, the feet in Novogen White, the thigh in ATAK-S, the heart and wing in ISA-Brown, CD-2 percentages were higher than in CD-1. 
Table 1 Hen slaughtering performance

\begin{tabular}{|c|c|c|c|c|c|c|}
\hline Variables & & Novogen White & ATAK-S & ISA-Brown & $P$-value & Strains combined \\
\hline \multirow{4}{*}{ Body weight (g) } & CD-1 & $1613.22 \pm 31.48^{b}$ & $1911.11 \pm 44.78^{a}$ & $1911.11 \pm 117.85^{a}$ & 0.013 & $1811.81 \pm 49.90$ \\
\hline & CD-2 & $1589.44 \pm 51.56^{b}$ & $1858.89 \pm 86.73^{a}$ & $1821.67 \pm 63.48^{a b}$ & 0.022 & $1756.67 \pm 44.77$ \\
\hline & $P$-value & 0.699 & 0.600 & 0.514 & & 0.414 \\
\hline & Treatment combined & $1601.33 \pm 29.45^{b}$ & $1885.00 \pm 47.77^{a}$ & $1866.39 \pm 65.83^{a}$ & $P<0.001$ & $1784.24 \pm 33.52$ \\
\hline \multirow{4}{*}{ Hot carcass (\%) } & CD-1 & $59.75 \pm 0.41^{b}$ & $64.23 \pm 0.96^{\mathrm{a}}$ & $63.37 \pm 0.94^{\mathrm{a}}$ & 0.001 & $62.45 \pm 0.59$ \\
\hline & CD-2 & $59.47 \pm 1.17^{b}$ & $67.12 \pm 1.48^{a}$ & $64.21 \pm 1.07^{a}$ & 0.001 & $63.60 \pm 0.93$ \\
\hline & $P$-value & 0.827 & 0.120 & 0.563 & & 0.300 \\
\hline & Treatment combined & $59.61 \pm 0.60^{\mathrm{b}}$ & $65.67 \pm 0.93^{a}$ & $63.79 \pm 0.70^{\mathrm{a}}$ & $P<0.001$ & $63.03 \pm 0.55$ \\
\hline \multirow{4}{*}{ Cold carcass (\%) } & CD-1 & $59.70 \pm 0.41^{b}$ & $64.16 \pm 0.96^{\mathrm{a}}$ & $63.25 \pm 0.91^{\mathrm{a}}$ & 0.001 & $62.37 \pm 0.58$ \\
\hline & CD-2 & $59.37 \pm 1.17^{b}$ & $65.80 \pm 1.12^{\mathrm{a}}$ & $63.97 \pm 1.08^{a}$ & 0.001 & $63.05 \pm 0.82$ \\
\hline & $P$-value & 0.794 & 0.284 & 0.615 & & 0.504 \\
\hline & Treatment combined & $59.53 \pm 0.60^{\mathrm{b}}$ & $64.98 \pm 0.74^{\mathrm{a}}$ & $63.60 \pm 0.69 a$ & $P<0.001$ & $62.71 \pm 0.50$ \\
\hline \multirow{4}{*}{$\begin{array}{l}\text { Head } \\
(\%)\end{array}$} & CD-1 & $4.51 \pm 0.13^{\mathrm{a}}$ & $3.01 \pm 0.11^{b}$ & $3.15 \pm 0.13^{\mathrm{b}}$ & $P<0.001$ & $3.56 \pm 0.15$ \\
\hline & CD-2 & $4.50 \pm 0.14^{\mathrm{a}}$ & $3.25 \pm 0.17 \mathrm{~b}$ & $3.04 \pm 0.08^{b}$ & $P<0.001$ & $3.60 \pm 0.15$ \\
\hline & $P$-value & 0.943 & 0.257 & 0.509 & & 0.852 \\
\hline & Treatment combined & $4.51 \pm 0.09^{a}$ & $3.13 \pm 0.10^{\mathrm{b}}$ & $3.10 \pm 0.08^{b}$ & $P<0.001$ & $3.58 \pm 0.10$ \\
\hline \multirow{4}{*}{$\begin{array}{l}\text { Feet } \\
(\%)\end{array}$} & CD-1 & $2.89 \pm 0.08^{b}$ & $3.40 \pm 0.11^{\mathrm{a}}$ & $3.46 \pm 0.13^{\mathrm{a}}$ & 0.002 & $3.24 \pm 0.08$ \\
\hline & CD-2 & $3.14 \pm 0.08^{b}$ & $3.87 \pm 0.26^{\mathrm{a}}$ & $3.80 \pm 0.12^{\mathrm{a}}$ & 0.011 & $3.60 \pm 0.12$ \\
\hline & $P$-value & 0.040 & 0.119 & 0.066 & & 0.014 \\
\hline & Treatment combined & $3.01 \pm 0.06^{b}$ & $3.63 \pm 0.15^{a}$ & $3.63 \pm 0.09 a$ & $P<0.001$ & $3.43 \pm 0.07$ \\
\hline \multirow{4}{*}{$\begin{array}{l}\text { Heart } \\
(\%)\end{array}$} & CD-1 & $0.55 \pm 0.03$ & $0.50 \pm 0.02$ & $0.51 \pm 0.03$ & 0.409 & $0.52 \pm 0.02$ \\
\hline & CD-2 & $0.53 \pm 0.04$ & $0.56 \pm 0.03$ & $0.58 \pm 0.02$ & 0.508 & $0.56 \pm 0.02$ \\
\hline & $P$-value & 0.781 & 0.081 & 0.052 & & 0.077 \\
\hline & Treatment combined & $0.54 \pm 0.02$ & $0.53 \pm 0.02$ & $0.55 \pm 0.02$ & 0.831 & $0.54 \pm 0.01$ \\
\hline \multirow{4}{*}{$\begin{array}{l}\text { Liver } \\
(\%)\end{array}$} & CD-1 & $2.51 \pm 0.18$ & $2.22 \pm 0.12$ & $2.27 \pm 0.12$ & 0.311 & $2.33 \pm 0.08$ \\
\hline & $\mathrm{CD}-2$ & $2.31 \pm 0.11$ & $2.08 \pm 0.07$ & $2.28 \pm 0.15$ & 0.285 & $2.22 \pm 0.06$ \\
\hline & $P$-value & 0.368 & 0.300 & 0.957 & & 0.307 \\
\hline & Treatment combined & $2.42 \pm 0.11$ & $2.15 \pm 0.07$ & $2.28 \pm 0.09$ & 0.117 & $2.28 \pm 0.05$ \\
\hline
\end{tabular}


Ylldırım et al. / GSC Biological and Pharmaceutical Sciences 2018, 05(03), 054-065

\begin{tabular}{|c|c|c|c|c|c|c|}
\hline \multirow{4}{*}{$\begin{array}{l}\text { Gizzard } \\
(\%)\end{array}$} & CD-1 & $2.49 \pm 0.12$ & $2.28 \pm 0.09$ & $2.24 \pm 0.10$ & 0.213 & $2.34 \pm 0.06$ \\
\hline & CD-2 & $2.51 \pm 0.12$ & $2.35 \pm 0.15$ & $2.15 \pm 0.19$ & 0.274 & $2.34 \pm 0.09$ \\
\hline & $P$-value & 0.897 & 0.704 & 0.696 & & 0.987 \\
\hline & Treatment combined & $2.50 \pm 0.08$ & $2.32 \pm 0.09$ & $2.19 \pm 0.11$ & 0.065 & $2.34 \pm 0.06$ \\
\hline \multirow{4}{*}{$\begin{array}{l}\text { Thigh } \\
(\%)\end{array}$} & CD-1 & $19.48 \pm 0.65$ & $20.13 \pm 0.41$ & $20.32 \pm 0.61$ & 0.555 & $19.98 \pm 0.32$ \\
\hline & CD-2 & $19.34 \pm 0.59^{b}$ & $22.77 \pm 0.87^{a}$ & $20.78 \pm 0.66^{\mathrm{ab}}$ & 0.009 & $20.96 \pm 0.48$ \\
\hline & $P$-value & 0.869 & 0.014 & 0.611 & & 0.095 \\
\hline & Treatment combined & $19.41 \pm 0.43^{\mathrm{b}}$ & $21.45 \pm 0.56^{\mathrm{a}}$ & $20.55 \pm 0.44^{\mathrm{ab}}$ & 0.016 & $20.47 \pm 0.30$ \\
\hline \multirow{4}{*}{$\begin{array}{l}\text { Breast } \\
(\%)\end{array}$} & CD-1 & $16.17 \pm 0.23^{b}$ & $17.69 \pm 0.41^{\mathrm{a}}$ & $16.53 \pm 0.42^{\mathrm{ab}}$ & 0.019 & $16.79 \pm 0.24$ \\
\hline & CD-2 & $16.16 \pm 0.52$ & $16.86 \pm 0.38$ & $16.11 \pm 0.74$ & 0.578 & $16.37 \pm 0.32$ \\
\hline & $P$-value & 0.983 & 0.161 & 0.623 & & 0.296 \\
\hline & Treatment combined & $16.16 \pm 0.27^{b}$ & $17.27 \pm 0.29 \mathrm{a}$ & $16.32 \pm 0.41^{\mathrm{ab}}$ & 0.045 & $16.58 \pm 0.20$ \\
\hline \multirow{4}{*}{$\begin{array}{l}\text { Wing } \\
(\%)\end{array}$} & CD-1 & $5.66 \pm 0.19^{a}$ & $6.55 \pm 0.13^{\mathrm{b}}$ & $6.20 \pm 0.20^{\mathrm{ab}}$ & 0.005 & $6.14 \pm 0.12$ \\
\hline & CD-2 & $5.77 \pm 0.18^{b}$ & $6.69 \pm 0.71^{b}$ & $7.77 \pm 0.35^{\mathrm{a}}$ & $P<0.001$ & $6.75 \pm 0.22$ \\
\hline & $P$-value & 0.670 & 0.614 & 0.001 & & 0.018 \\
\hline & Treatment combined & $5.72 \pm 0.13^{\mathrm{b}}$ & $6.62 \pm 0.13^{\mathrm{a}}$ & $6.98 \pm 0.27^{a}$ & $P<0.001$ & $6.44 \pm 0.13$ \\
\hline \multirow{4}{*}{$\begin{array}{l}\text { Spleen } \\
(\%)\end{array}$} & CD-1 & $0.11 \pm 0.01$ & $0.09 \pm 0.01$ & $0.11 \pm 0.01$ & 0.308 & $0.10 \pm 0.01$ \\
\hline & CD-2 & $0.11 \pm 0.01$ & $0.13 \pm 0.02$ & $0.11 \pm 0.01$ & 0.581 & $0.12 \pm 0.01$ \\
\hline & $P$-value & 0.663 & 0.110 & 0.738 & & 0.284 \\
\hline & Treatment combined & $0.11 \pm 0.01$ & $0.11 \pm 0.01$ & $0.11 \pm 0.01$ & 0.998 & $0.11 \pm 0.01$ \\
\hline \multirow{4}{*}{$\begin{array}{l}\text { Abdominal } \\
(\%)\end{array}$} & CD-1 & $2.42 \pm 0.22^{\mathrm{a}}$ & $4.12 \pm 0.53^{b}$ & $3.57 \pm 0.60^{\mathrm{ab}}$ & 0.054 & $3.37 \pm 0.30$ \\
\hline & CD-2 & $1.85 \pm 0.32$ & $3.44 \pm 0.55$ & $3.38 \pm 0.81$ & 0.120 & $2.89 \pm 0.36$ \\
\hline & $P$-value & 0.163 & 0.385 & 0.849 & & 0.309 \\
\hline & Treatment combined & $2.13 \pm 0.20^{\mathrm{b}}$ & $3.78 \pm 0.38^{a}$ & $3.48 \pm 0.49^{a}$ & 0.007 & $3.13 \pm 0.23$ \\
\hline \multirow{4}{*}{$\begin{array}{l}\text { Neck and back } \\
(\%)\end{array}$} & CD-1 & $18.28 \pm 0.81$ & $18.62 \pm 1.13$ & $19.59 \pm 1.01$ & 0.636 & $18.83 \pm 0.56$ \\
\hline & CD-2 & $17.58 \pm 0.76$ & $18.27 \pm 1.62$ & $18.77 \pm 0.79$ & 0.761 & $18.21 \pm 0.63$ \\
\hline & $P$-value & 0.537 & 0.860 & 0.532 & & 0.464 \\
\hline & Treatment combined & $17.94 \pm 0.55$ & $18.45 \pm 0.96$ & $19.18 \pm 0.63$ & 0.491 & $18.52 \pm 0.42$ \\
\hline
\end{tabular}
same row for each section are significantly different $(\mathrm{P}<0.05)$ 


\subsection{Blood analyses of hens}

The results of biochemical tests for MDA, GSH, SOD, GPx and CAT are shown in Table 2. When statistically significant differents are examined in the treatment combined groups, the MDA data of ISA-Brown hens were lower than the Novogen White and ATAK-S hens, but the SOD and GPx variables of ISA-Brown hens were higher than other strains. In the strain combined groups, all of the CD-1 variables except for the MDA received the highest value compared to CD- 2 . In addition, when the results between CD-1 and CD- 2 in itself of the strains were examined, in all of other data except for the GPX of ATAK-S and ISA-Brown significant differences were found. As a result, when the stress parameters were evaluated, chickens in CD-1 experienced more stress than CD-2. In addition, it may be said that the ISA-Brown strain is more resistant to stress than the other strains.

Table 2 Flock biochemical results

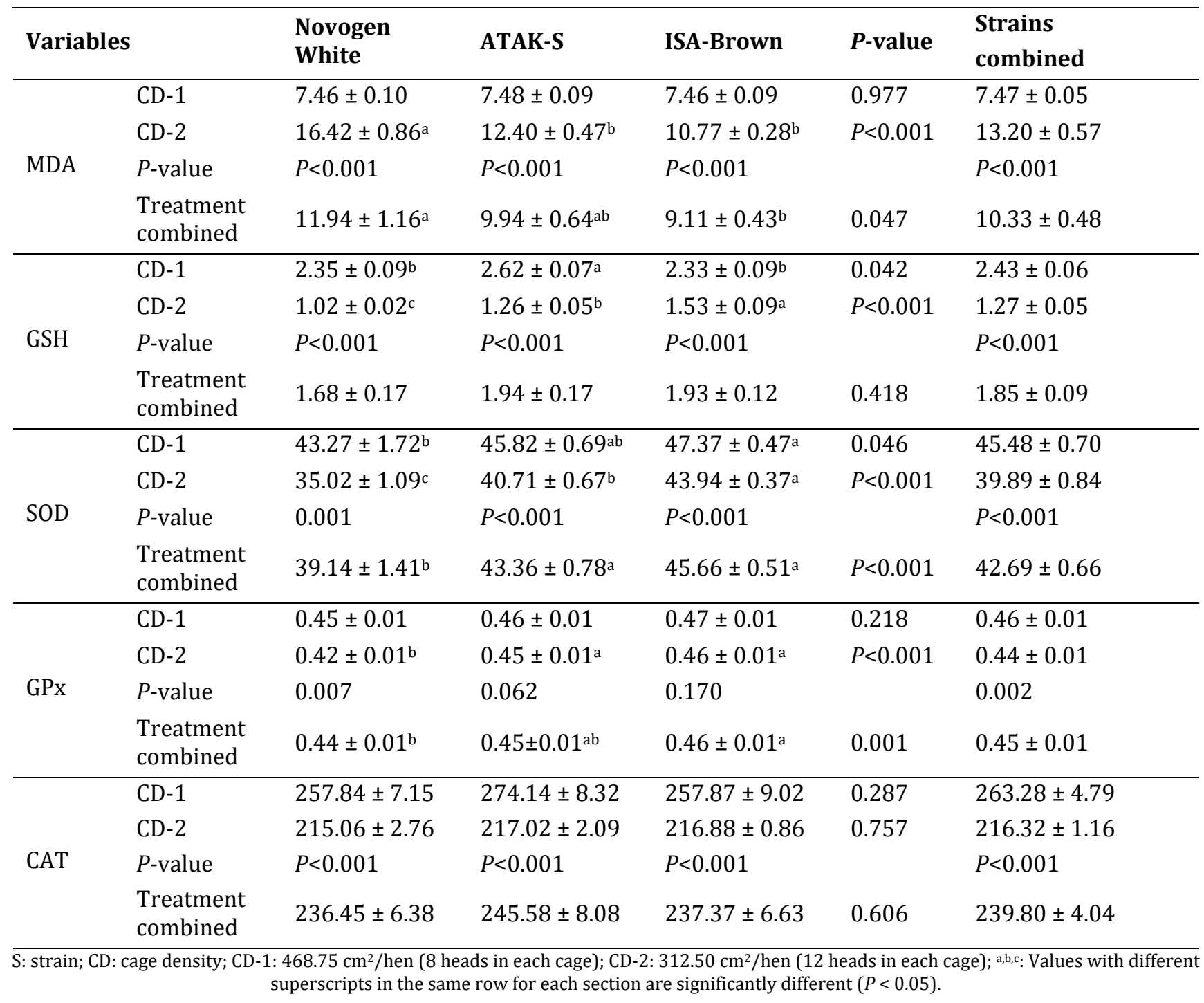

\subsection{Meat quality of hens}

The results related to meat quality of hens used in the study are presented in Table 3 . In the pH, aw, TBARS and colour $\left(\mathrm{L}^{*}, \mathrm{a}^{*}, \mathrm{~b}^{*}\right)$ parameters of hens meat samples were not detected a significant difference among the strains combined $(P$ $>0.05)$. However, the water activity in the treatment combined was highest in ISA-Brown strain $(P<0.05)$. In terms of TBARS, Novogen White strain in CD-2 cages and ISA-Brown strain in CD-1 cages received the lowest value $(P<0.05)$. When the color parameters were examined, yellowness $\left(b^{*}\right)$ was observed in the lowest Novogen White strain $(P<$ 0.05). When the $\mathrm{pH}$, aw, TBARS and color parameters are considered as a whole, it may be said that ISA-Brown strain meats in CD-1 cages are better than others. 
Table 3 The effects of housing environment on laying hen on $\mathrm{pH}$, water activity, TBARS and colour parameters (L*, a*, $\mathrm{b}^{*}$ ) in chicken breast meat

\begin{tabular}{|c|c|c|c|c|c|c|}
\hline Variables & & $\begin{array}{l}\text { Novogen } \\
\text { White }\end{array}$ & ATAK-S & ISA-Brown & $P$-value & $\begin{array}{l}\text { Strains } \\
\text { combined }\end{array}$ \\
\hline \multirow{4}{*}{$\mathrm{pH}$} & CD-1 & $5.74 \pm 0.17$ & $5.76 \pm 0.15$ & $5.76 \pm 0.17$ & 0.996 & $5.75 \pm 0.09$ \\
\hline & CD-2 & $5.70 \pm 0.15$ & $5.80 \pm 0.13$ & $5.74 \pm 0.15$ & 0.887 & $5.75 \pm 0.08$ \\
\hline & $P$-value & 0.859 & 0.836 & 0.904 & & 0.941 \\
\hline & $\begin{array}{l}\text { Treatment } \\
\text { combined }\end{array}$ & $5.72 \pm 0.11$ & $5.78 \pm 0.10$ & $5.75 \pm 0.11$ & 0.930 & $5.75 \pm 0.06$ \\
\hline \multirow{4}{*}{$\begin{array}{l}\text { Water } \\
\text { activity }\end{array}$} & CD-1 & $0.9923 \pm 0.002$ & $0.9956 \pm 0.001$ & $0.9971 \pm 0.001$ & 0.102 & $0.9950 \pm 0.001$ \\
\hline & CD-2 & $0.9936 \pm 0.002$ & $0.9972 \pm 0.002$ & $0.9966 \pm 0.001$ & 0.256 & $0.9958 \pm 0.001$ \\
\hline & $P$-value & 0.667 & 0.404 & 0.659 & & 0.545 \\
\hline & $\begin{array}{l}\text { Treatment } \\
\text { combined }\end{array}$ & $0.9930 \pm 0.002^{b}$ & $0.9964 \pm 0.001^{\mathrm{ab}}$ & $0.9970 \pm 0.001^{\mathrm{a}}$ & 0.027 & $0.9954 \pm 0.001$ \\
\hline \multirow{4}{*}{ TBARS } & CD-1 & $2.90 \pm 0.18^{a}$ & $3.08 \pm 0.55^{\mathrm{a}}$ & $1.64 \pm 0.12^{b}$ & 0.017 & $2.53 \pm 0.24$ \\
\hline & CD-2 & $1.67 \pm 0.12^{b}$ & $2.91 \pm 0.47^{a}$ & $3.61 \pm 0.24^{\mathrm{a}}$ & 0.002 & $2.73 \pm 0.26$ \\
\hline & $P$-value & $P<0.001$ & 0.826 & $P<0.001$ & & 0.590 \\
\hline & $\begin{array}{l}\text { Treatment } \\
\text { combined }\end{array}$ & $2.28 \pm 0.21$ & $3.00 \pm 0.34$ & $2.62 \pm 0.32$ & 0.258 & $2.63 \pm 0.18$ \\
\hline \multirow{4}{*}{$\mathrm{L}^{*}$} & CD-1 & $55.18 \pm 1.15$ & $54.02 \pm 2.46$ & $56.90 \pm 1.53$ & 0.591 & $55.36 \pm 1.02$ \\
\hline & $\mathrm{CD}-2$ & $53.68 \pm 2.24$ & $53.24 \pm 1.39$ & $51.30 \pm 2.22$ & 0.673 & $52.74 \pm 1.11$ \\
\hline & $P$-value & 0.585 & 0.789 & 0.058 & & 0.090 \\
\hline & $\begin{array}{l}\text { Treatment } \\
\text { combined }\end{array}$ & $54.40 \pm 2.24$ & $53.63 \pm 1.35$ & $54.15 \pm 1.42$ & 0.925 & $54.05 \pm 0.77$ \\
\hline \multirow{4}{*}{$a^{*}$} & CD-1 & $2.37 \pm 0.75$ & $4.64 \pm 1.81$ & $3.26 \pm 0.85$ & 0.282 & $3.42 \pm 0.54$ \\
\hline & CD-2 & $3.21 \pm 0.83$ & $4.79 \pm 1.11$ & $3.73 \pm 0.63$ & 0.453 & $3.91 \pm 0.50$ \\
\hline & $P$-value & 0.591 & 0.928 & 0.500 & & 0.520 \\
\hline & $\begin{array}{l}\text { Treatment } \\
\text { combined }\end{array}$ & $2.89 \pm 0.59$ & $4.71 \pm 0.75$ & $3.35 \pm 0.49$ & 0.114 & $3.67 \pm 0.37$ \\
\hline \multirow{4}{*}{$b^{*}$} & CD-1 & $1.25 \pm 0.26^{b}$ & $3.58 \pm 0.57^{a}$ & $2.48 \pm 0.59^{a b}$ & 0.019 & $2.44 \pm 0.36$ \\
\hline & CD-2 & $2.28 \pm 0.72$ & $2.22 \pm 0.83$ & $2.41 \pm 0.85$ & 0.985 & $2.31 \pm 0.44$ \\
\hline & $P$-value & 0.220 & 0.208 & 0.955 & & 0.817 \\
\hline & $\begin{array}{l}\text { Treatment } \\
\text { combined }\end{array}$ & $1.77 \pm 0.43$ & $2.90 \pm 1.81$ & $2.39 \pm 0.46$ & 0.273 & $2.37 \pm 0.77$ \\
\hline
\end{tabular}

S: strain; CD: cage density; CD-1: $468.75 \mathrm{~cm}^{2} /$ hen (8 heads in each cage); CD-2: $312.50 \mathrm{~cm}^{2} /$ hen (12 heads in each cage); a,b: Values with different superscripts in the same row for each section are significantly different $(P<0.05)$. NS: Not significant $(P>0.05)$; ${ }^{*} P<0.05$; ${ }^{* *}: P<0.01$.

The microbiological results of hens meat used in the experiment are shown in Table 4 . When the microbiological results of the breast meat were examined, a significant difference was observed only in Enterobacteriaceae counts in strain combined $(P<0.05)$. As for the treatment combined, there were statistically significant differences between TAMB $(P<$ $0.05)$, TPAB $(P<0.001)$ and Coliforms $(P<0.05)$. In general, the Novogen White strain in CD-1 cages and ISA-Brown strain in CD-2 cages showed a lower bacterial count. Also, ATAK-S strain was different in both cages according to the type of bacteria. According to the bacterial data examined, it may be said that the ISA-Brow strain is superior to other strains in terms of cage density. 
Table 4 The effects of housing environment on laying hen on total aerobic mesophilic bacteria (TAMB), total aerobic psychrophilic bacteria (TAPB), Enterobacteriaceae, Coliforms, and Staphylococcus aureus counts in chicken breast meat

\begin{tabular}{|c|c|c|c|c|c|c|}
\hline Variables & & $\begin{array}{l}\text { Novogen } \\
\text { White }\end{array}$ & ATAK-S & ISA-Brown & $P$-value & $\begin{array}{l}\text { Strains } \\
\text { combined }\end{array}$ \\
\hline \multirow{4}{*}{ TAMB } & CD-1 & $2.99 \pm 0.29 c$ & $3.79 \pm 0.23^{b}$ & $5.12 \pm 0.10^{\mathrm{a}}$ & 0.002 & $3.97 \pm 0.33$ \\
\hline & $\mathrm{CD}-2$ & $4.40 \pm 0.20$ & $4.09 \pm 0.26$ & $4.41 \pm 0.15$ & 0.503 & $4.30 \pm 0.12$ \\
\hline & $P$-value & 0.016 & 0.043 & 0.017 & & 0.349 \\
\hline & $\begin{array}{l}\text { Treatment } \\
\text { combined }\end{array}$ & $3.70 \pm 0.35^{b}$ & $3.94 \pm 0.17 \mathrm{ab}$ & $4.77 \pm 0.18^{a}$ & 0.021 & $4.13 \pm 0.18$ \\
\hline \multirow{4}{*}{ ТРАВ } & $\mathrm{CD}-1$ & $2.23 \pm 0.12^{b}$ & $3.33 \pm 0.01^{\mathrm{a}}$ & $2.24 \pm 0.04^{b}$ & $P<0.001$ & $2.60 \pm 0.18$ \\
\hline & $\mathrm{CD}-2$ & $2.42 \pm 0.03^{b}$ & $3.40 \pm 0.06^{\mathrm{a}}$ & $2.26 \pm 0.03^{c}$ & $P<0.001$ & $2.69 \pm 0.18$ \\
\hline & $P$-value & 0.196 & 0.254 & 0.732 & & 0.718 \\
\hline & $\begin{array}{l}\text { Treatment } \\
\text { combined }\end{array}$ & $2.33 \pm 0.07^{b}$ & $3.36 \pm 0.03^{a}$ & $2.25 \pm 0.02^{\mathrm{b}}$ & $P<0.001$ & $2.65 \pm 0.13$ \\
\hline \multirow{4}{*}{ Enterobacteriaceae } & CD-1 & $2.77 \pm 0.04^{b}$ & $2.68 \pm 0.12^{b}$ & $3.18 \pm 0.02^{\mathrm{a}}$ & 0.008 & $2.88 \pm 0.18$ \\
\hline & $\mathrm{CD}-2$ & $3.14 \pm 0.27$ & $3.44 \pm 0.16$ & $2.98 \pm 0.01$ & 0.261 & $3.19 \pm 0.11$ \\
\hline & $P$-value & 0.253 & 0.020 & 0.001 & & 0.045 \\
\hline & $\begin{array}{l}\text { Treatment } \\
\text { combined }\end{array}$ & $2.96 \pm 0.15$ & $3.06 \pm 0.19$ & $3.08 \pm 0.05$ & 0.809 & $3.03 \pm 0.11$ \\
\hline \multirow{4}{*}{ Coliforms } & $\mathrm{CD}-1$ & $2.43 \pm 0.30$ & $3.23 \pm 0.12$ & $2.90 \pm 0.03$ & 0.059 & $2.86 \pm 0.15$ \\
\hline & $\mathrm{CD}-2$ & $3.99 \pm 0.35^{\mathrm{a}}$ & $2.75 \pm 0.23^{\mathrm{b}}$ & $2.86 \pm 0.04^{b}$ & 0.020 & $3.20 \pm 0.23$ \\
\hline & $P$-value & 0.027 & 0.133 & 0.460 & & 0.227 \\
\hline & $\begin{array}{l}\text { Treatment } \\
\text { combined }\end{array}$ & $3.21 \pm 0.41$ & $2.99 \pm 0.16$ & $2.88 \pm 0.03$ & 0.644 & $3.03 \pm 0.40$ \\
\hline \multirow{4}{*}{ Staphylococcus aureus } & CD-1 & $2.06 \pm 0.06^{c}$ & $3.35 \pm 0.03^{\mathrm{a}}$ & $2.57 \pm 0.07^{b}$ & $P<0.001$ & $2.66 \pm 0.19$ \\
\hline & CD-2 & $2.93 \pm 0.05^{\mathrm{a}}$ & $2.43 \pm 0.22^{b}$ & $2.32 \pm 0.09 \mathrm{~b}$ & 0.045 & $2.56 \pm 0.12$ \\
\hline & $P$-value & $P<0.001$ & 0.014 & 0.080 & & 0.650 \\
\hline & $\begin{array}{l}\text { Treatment } \\
\text { combined }\end{array}$ & $2.49 \pm 0.20$ & $2.89 \pm 0.23$ & $2.45 \pm 0.08$ & 0.185 & $2.61 \pm 0.11$ \\
\hline
\end{tabular}

S: strain; CD: cage density; CD-1: $468.75 \mathrm{~cm}^{2} /$ hen (8 heads in each cage); CD-2: $312.50 \mathrm{~cm}^{2} /$ hen (12 heads in each cage); ${ }^{\text {ab,c: }}$ Values with different superscripts in the same row for each section are significantly different $(P<0.05)$. NS: Not significant $(P>0.05)$; ${ }^{*} P<0.05$; ${ }^{* *}: P<0.01$.

The statistical analyses of various data recorded for textural parameters of breast meat samples are observed in Table 5. The hardness, cohesiveness, adhesiveness, springiness, gumminess and chewiness of chicken meat samples were not found a significant differences among the strains combined textural analyses results $(P>0.05)$. But, when the significant different between strains in itself of the CD-1 and CD-2 were examined, statistically significant differences were observed. Besides, the Novogen White strain exhibited the lowest hardness, cohesiveness, adhesiveness, gumminess and chewiness breast meat samples in the treatment combined. Therefore, it may be said that the texture analyses results of the Novogen White strain are better than ISA-Brown and ATAK-S strains. 
Table 5 Textural parameters of breast meat

\begin{tabular}{|c|c|c|c|c|c|c|}
\hline Variables & & $\begin{array}{l}\text { Novogen } \\
\text { White }\end{array}$ & ATAK-S & ISA-Brown & $P$-value & $\begin{array}{l}\text { Strains } \\
\text { combined }\end{array}$ \\
\hline \multirow{4}{*}{ Hardness (N) } & CD-1 & $15.17 \pm 3.45^{b}$ & $29.21 \pm 3.41^{a}$ & $18.45 \pm 1.37^{b}$ & 0.011 & $21.11 \pm 2.14$ \\
\hline & CD-2 & $16.07 \pm 4.15$ & $19.29 \pm 3.11$ & $20.27 \pm 2.23$ & 0.643 & $18.54 \pm 1.82$ \\
\hline & $P$-value & 0.870 & 0.057 & 0.628 & & 0.367 \\
\hline & $\begin{array}{l}\text { Treatment } \\
\text { combined }\end{array}$ & $15.62 \pm 2.57^{b}$ & $24.25 \pm 2.66^{\mathrm{a}}$ & $19.61 \pm 1.26^{\mathrm{ab}}$ & 0.037 & $19.83 \pm 1.26$ \\
\hline \multirow{4}{*}{ Cohesiveness } & CD-1 & $0.28 \pm 0.02^{\mathrm{b}}$ & $0.35 \pm 0.03^{\mathrm{ab}}$ & $0.38 \pm 0.02^{\mathrm{a}}$ & 0.026 & $0.34 \pm 0.02$ \\
\hline & CD-2 & $0.32 \pm 0.05^{b}$ & $0.35 \pm 0.02^{\mathrm{ab}}$ & $0.44 \pm 0.01^{\mathrm{a}}$ & 0.026 & $0.37 \pm 0.02$ \\
\hline & $P$-value & 0.404 & 0.956 & 0.029 & & 0.158 \\
\hline & $\begin{array}{l}\text { Treatment } \\
\text { combined }\end{array}$ & $0.30 \pm 0.02^{\mathrm{b}}$ & $0.35 \pm 0.02^{\mathrm{ab}}$ & $0.41 \pm 0.02^{\mathrm{a}}$ & 0.001 & $0.35 \pm 0.01$ \\
\hline \multirow{4}{*}{ Adhesiveness } & CD-1 & $-1.38 \pm 0.17^{a b}$ & $-1.93 \pm 0.44^{\mathrm{b}}$ & $-0.87 \pm 0.08^{a}$ & 0.050 & $-1.40 \pm 0.18$ \\
\hline & $\mathrm{CD}-2$ & $-1.12 \pm 0.31$ & $-1.98 \pm 0.51$ & $-0.82 \pm 0.14$ & 0.087 & $-1.30 \pm 1.78$ \\
\hline & $P$-value & 0.477 & 0.952 & 0.731 & & 0.752 \\
\hline & $\begin{array}{l}\text { Treatment } \\
\text { combined }\end{array}$ & $-1.25 \pm 0.17^{\mathrm{a}}$ & $-1.95 \pm 0.32^{b}$ & $-0.85 \pm 0.08^{a}$ & 0.003 & $-1.35 \pm 0.14$ \\
\hline \multirow{4}{*}{$\begin{array}{l}\text { Springiness } \\
(\mathrm{mm})\end{array}$} & CD-1 & $0.66 \pm 0.04^{\mathrm{a}}$ & $0.51 \pm 0.04^{\mathrm{b}}$ & $0.49 \pm 0.03^{\mathrm{b}}$ & 0.011 & $0.55 \pm 0.03$ \\
\hline & CD-2 & $0.56 \pm 0.06$ & $0.58 \pm 0.05$ & $0.44 \pm 0.03$ & 0.121 & $0.53 \pm 0.03$ \\
\hline & $P$-value & 0.215 & 0.303 & 0.303 & & 0.565 \\
\hline & $\begin{array}{l}\text { Treatment } \\
\text { combined }\end{array}$ & $0.61 \pm 0.04^{\mathrm{a}}$ & $0.55 \pm 0.03^{\mathrm{ab}}$ & $0.46 \pm 0.02^{\mathrm{b}}$ & 0.009 & $0.54 \pm 0.02$ \\
\hline \multirow{4}{*}{ Gumminess (N) } & $\mathrm{CD}-1$ & $4.58 \pm 1.14^{b}$ & $10.13 \pm 0.97^{a}$ & $7.112 \pm 0.69^{a b}$ & 0.003 & $7.28 \pm 0.75$ \\
\hline & CD-2 & $5.81 \pm 1.73$ & $6.80 \pm 1.30$ & $8.93 \pm 0.89$ & 0.277 & $7.18 \pm 5.50$ \\
\hline & $P$-value & 0.567 & 0.069 & 0.143 & & 0.928 \\
\hline & $\begin{array}{l}\text { Treatment } \\
\text { combined }\end{array}$ & $5.20 \pm 1.00^{\mathrm{b}}$ & $8.46 \pm 0.92^{\mathrm{a}}$ & $8.03 \pm 0.60^{\mathrm{a}}$ & 0.023 & $7.23 \pm 0.54$ \\
\hline \multirow{4}{*}{$\begin{array}{l}\text { Chewiness } \\
\text { (Nxmm) }\end{array}$} & CD-1 & $3.22 \pm 0.92$ & $5.17 \pm 0.65$ & $3.44 \pm 0.36$ & 0.121 & $3.95 \pm 0.43$ \\
\hline & $\mathrm{CD}-2$ & $3.34 \pm 0.97$ & $4.15 \pm 1.08$ & $3.98 \pm 0.50$ & 0.795 & $3.82 \pm 0.49$ \\
\hline & $P$-value & 0.934 & 0.440 & 0.408 & & 0.850 \\
\hline & $\begin{array}{l}\text { Treatment } \\
\text { combined }\end{array}$ & $3.28 \pm 0.64$ & $4.66 \pm 0.62$ & $3.71 \pm 0.31$ & 0.200 & $3.89 \pm 0.32$ \\
\hline
\end{tabular}

\section{Discussion}

The different response in terms of hen slaughtering performance and body weight observed among the different genotypes is related to their genetic assessment [9]. Similarly, in this study, the body weights and carcass traits of three different genotypes founded differences between genotypes. The ISA-Brown and ATAK-S are genotypically larger strains than Novogen White and, therefore, they have higher values in body weight and many carcass traits. This situation is thought to be the reflection to phenotypic parameters of genotypic differences between strains. 
Some researchers reported that reduced hen body weight in intermediate group size compared to large and small group sizes, which they attributed to social intolerance [25]. Besides, the cage density required for the wingspan of the commercial laying hen hybrids used today is not sufficient in the existing cage systems [26]. In this study, when the effect of cage density on carcass traits was examined, it was observed that CD-1 values, which are wide density, were higher than CD-2 at a significant level, especially in the feet and wings weights. As a consequence, this situation might be an indication of more affected by the cage density of the organs that are more contact with the cage of hens.

Stress leads to increasing whole body metabolism and therefore depletes muscle glycogen stores and stimulates protein catabolism, affecting body weight [2]. As seen in this study, the conventional cages have been shown many times to limit the natural movements of animals, to cause fear and stress [26]. In addition, the high density of chicken in CD-2 cages generally caused to have higher stress parameters than those in CD-1 cages. When examined in terms of strains, ISABrown, ATAK-S and Novogen White formed the order of resistance to stress, respectively. If breeders are forced to raise more animals in a cage, it may be said that the ISA-Brown strain is more resistant to the incidence of stress than the other strains here.

In the present study, the $\mathrm{pH}$ values of breast meat did not differ between strains. $(\mathrm{P}>0.05)$. However, in the previous studies conducted on different genotypes, the $\mathrm{pH}$ values were different between strains [2, 9]. Also, the $\mathrm{pH}$ value (6.87 $\mathrm{pH}$ ) of hen's breast meat stated by Altun and Atasever [27] was higher than the data in this study. The results concerning water activity in this study were higher than the water activity of hens breast meat average value $(0.8890$ aw) stated by other researchers [27]. The meat colour could be affected by the movement of the hens [9]. However, in this study, the different cage density was not affected the meat colour of hens. $(P<0.05)$. Only, the yellowness $\left(b^{*}\right)$ in the CD-1 cages showed a difference between strains. The results concerning TAMB and TPAB in this study were lower than the average data of hen's breast meat stated by Altun and Atasever [27]. The Coliforms and Staphylococcus aureus counts in chicken breast meat presented this study was similar to that reported by Efe and Gümüşsoy [28], but the Enterobacteriaceae count was higher than data reported by same researchers.

Hardness, cohesiveness, chewiness, and springiness are important parameters for the evaluation of meat textures, and, in particular, the value of cohesiveness and hardness has been recognized as an important indicator of the textural properties of the meat [29]. In this experiment, the Novogen White strain was exhibited the best results in terms of the value of cohesiveness and hardness. In some studies conducted in breast meats of laying hens with different genotypes, the authors reported that Hy-Line White presented the lowest tenderness, chewiness, and adhesivity, whereas the HyLine Brown showed the highest juiciness and solubility [9].

\section{Conclusions}

The effect of cage densities on carcass traits of hens was not found in many data, but many differences were observed due to genetic differences between strains. In particular, ISA-Brown and ATAK-S strains received higher values than the Novogen White strain in terms of carcass traits. Also, according to stress parameters, it was observed that ISA-Brown strain was more resistant than others in high cage density. Moreover, the bacterial count and meat quality results of hens, the ISA-Brown strain is better than other strains. However, the texture analyses results of the Novogen White strain are better than ISA-Brown and ATAK-S strains. As a result, the meat quality of the laying hens can be affected at different levels depending on the genetic structure of the chickens and the cage density of which they are reared. So, the further research is needed to determine meat quality of hens of these genotypes in a different phase of the laying cycle and in a different cage density.

\section{Compliance with ethical standards}

\section{Acknowledgments}

The research was founded by Ataturk University Scientific Research Projects, Erzurum, Turkey (SRP-2016/71).

\section{Disclosure of conflict of interest}

The authors declare that they have no conflict of interests.

\section{Statement of ethical approval}

This research was carried out in accordance with the guidelines of the Atatürk University Local Board of Ethics Committee for Animal Experiments, which has approved the study protocol of this research (HADYEK decision no: 72). 


\section{References}

[1] Cravener TL, Roush WB and Mashaly MM. (1992). Broiler production under varying population densities. Poult. Sci. 71, 427-433.

[2] Frizzell K.M, Lynch E, Rathgeber BM, Dixon WT, Putman CT and Jendral MJ. (2017). Effect of housing environment on laying hen meat quality: Assessing Pectoralis major $\mathrm{pH}$, colour and tenderness in three strains of 80-81 weekold layers housed in conventional and furnished cages. Br. Poult. Sci. 58, 50-58.

[3] Bendall JR. (1973). Postmortem changes in muscle. In: G. Bourne (Editor). The Structure and Function of Muscle. Academic Press. New York, (US), 244-309.

[4] Van Laack RLJM, Liu CH, Smith MO and Loveday HD. (2000). Characteristics of pale, soft, exudative broiler breast meat. Poult. Sci. 79, 1057-1061.

[5] Quio M, Fletcher DL, Smith DP and Northcutt JK. (2001). The effect of broiler breast meat color on pH, moisture, water-holding capacity, and emulsification capacity. Poult. Sci. 80, 676-680.

[6] Barbut S, Zhang L and Marcone M. (2005). Effects of pale, normal, and dark chicken breast meat on microstructure, extractable proteins, and cooking of marinated fillets. Poult. Sci. 84: 797-802.

[7] Ahn DU and Maurer AJ. (1990). Poultry meat color: pH and the heme-complex forming reaction. Poult. Sci. 69, 2040-2050.

[8] Damme K and Ristic M. (2003). Fattening performance, meat yield and economic aspects of meat and layer type hybrids. Worlds Poult. Sci. J. 59, 50-53.

[9] Rizzi C, Marangon A and Chiericato GM. (2007). Effect of genotype on slaughtering performance and meat physical and sensory characteristics of organic laying hens. Poult. Sci. 86, 128-135.

[10] Gregory NG and Wilkins LJ. (1989). Broken bones in domestic fowl: handling and processing damage in end-oflay battery hens. Br. Poult. Sci. 30, 555-562.

[11] Hocquette JF, Gondret F, Baéza E, Médale F, Jurie C and Pethick DW. (2010). Intramuscular fat content in meatproducing animals: development, genetic and nutritional control, and identification of putative markers. Animal. 4, 303-319.

[12] Atasoy F and Aksoy T. (2005). The effects of sex separate rearing and early feed restriction of broiler chicks on carcass weight and valuable cuts. Vet. J. Ankara Univ. 52, 53-56.

[13] Seven PT, Seven I, Yılmaz M and Şimşek ÜG. (2008). The effects of Turkish propolis on growth and carcass characteristics in broilers under heat stress. Anim. Feed Sci. Technol. 146, 137-148.

[14] Yildirim F., Tunç, M. A. (2018). The effect of dietary tarragon (Artemisia Dracunculus) powders in different levels on carcass characteristics and some internal organ's weight of broiler chickens. Rev. Bras. Cienc. Avic. 20, 179182.

[15] Sisein EA. (2014). Biochemistry of free radicals and antioxidants. Sch. Acad. J. Biosci. 2, 110-118.

[16] Yoshioka T, Kawada K, Shimada T and Mori M. (1979). Lipid peroxidation in maternal and cord blood and protective mechanism against activated-oxygen toxicity in the blood. Am. J. Obstet. Gynecol. 135, 372-376.

[17] Tietze F. (1969). Enzymic method for quantitative determination of nanogram amounts of total and oxidized glutathione: applications to mammalian blood and other tissues. Anal. Biochem. 27, 502-522.

[18] Sun YI, Oberley LW and Li Y. (1988). A simple method for clinical assay of superoxide dismutase. Clin. Chem. 34, 497-500.

[19] Matkovics B. (1988). Determination of enzyme activity in lipid peroxidation and glutathione pathways. Laboratoriumi Diagnosztika. 15, 248-50.

[20] Goth L. (1991). A simple method for determination of serum catalase activity and revision of reference range. Clin. Chim. Acta. 196, 143-51.

[21] Gökalp HY, Kaya M, Tülek Y and Zorba 0. (2001). Guide for quality control and laboratory application of meat products. No: 751. Atatürk University Press. Erzurum (TR). 
[22] Gümüş R., Gelen S.U., Köseoğlu S., Ozkanlar S., Ceylan Z. G., Imik, H., (2018). The effects of fucoxanthin dietary inclusion on the growth performance, antioxidant metabolism and meat quality of broilers. Braz. J. Poult. Sci. 20, 487-496.

[23] Baumgart J, Becker B and Stephan. (1986). Mikrobiologische Untersuchung von Lebensmitteln. Hamburg, Germany, Behr's Verlag.

[24] Kruk ZA, Yun H, Rutley DL, Lee EJ, Kim YJ and Jo C. (2011). The effect of high pressure on microbial population, meat quality and sensory characteristics of chicken breast fillet. Food Control. 22, 6-12.

[25] Keeling LJ, Estevez I, Newberry RC and Correia MG. (2003). Production-related traits of layers reared in different sized flocks: the concept of problematic intermediate group sizes. Poult. Sci. 82, 1393-1396.

[26] Bozkurt Z. (2009). Laying hen welfare in battery cages and alternative systems. Kocatepe Vet. J. 2, 59-67.

[27] Altun SK and Atasever M. (2018). Investigation of Some Pathogenic Bacteria of Chicken Meat Presented for Sale in Erzurum. Manas J. Agr. Vet. Life Sci. 8, 36-50.

[28] Efe M and Gümüşsoy KS. (2005). Microbiological analysis of chicken meat served for consumption in Ankara garrison. J. Health Sci. 14, 151-157.

[29] Salakova A, Strakova E, Valkova V, Buchtova H and Steinhauserova I. (2009). Quality indicators of chicken broiler raw and cooked meat depending on their sex, Acta Vet. Brno. 78, 497-504.

\section{How to cite this article}

Yıldırım F, Özentük U, Özlü H, Urçar Gelen S, Apaydın Yıldırım B, Atasever M and Yıldız A. (2018). Effect of genotype on slaughtering performance, blood analyses and meat quality of laying hens reared in different conventional cage densities. GSC Biological and Pharmaceutical Sciences, 5(3), 54-65. 